\title{
Drug-Induced Interstitial Pneumonia due to Application of FOLFOX as Adjuvant Chemotherapy after Rectal Cancer Surgery: A Case Report and Literature Review
}

\author{
Senichiro Yanagawa ${ }^{a}$ Nozomi Karakuchi ${ }^{a}$ Tetsuya Mochizukic \\ Shinya Kodama ${ }^{a}$ Yukio Takeshima ${ }^{b}$ Kazuo Sumimoto ${ }^{a, c}$ \\ aDepartment of Surgery, Yoshida General Hospital, Akitakata City, Japan; ${ }^{b}$ Department \\ of Pathology, Graduate School of Biomedical and Health Sciences, Hiroshima University, \\ Hiroshima, Japan; 'Department of Gastroenterological and Transplant Surgery, Graduate \\ School of Biomedical and Health Sciences, Hiroshima University, Hiroshima, Japan
}

\section{Keywords}

FOLFOX · Drug-induced pneumonia - Rectal cancer · Steroid therapy

\begin{abstract}
The regimen of oxaliplatin with 5-fluorouracil plus I-leucovorin (FOLFOX) has become one of the most commonly used first-line chemotherapy for patients with advanced colorectal cancer and it provides an increase in disease-free survival as well as an overall survival benefit. Although FOLFOX chemotherapy has helped to improve the clinical outcomes in these patients, the regimen is associated with some therapeutic issues or uncontrolled side effects. Gastrointestinal, neurosensory, and hematological toxicities have frequently been observed in patients treated with FOLFOX, and consequently, some palliative treatment has been established to combat such complications. However, pulmonary toxicities including drug-induced interstitial pneumonia (DI-IP) is rarely observed in these patients and a curative treatment is yet to be established. DI-IP due to chemotherapy is most commonly observed in patients treated with mitomycin, paclitaxel, docetaxel, or gemcitabine. Steroid therapy is mostly used to treat DI-IP, although the efficacy of such treatments is not supported with adequate evidence. FOLFOX-induced interstitial pneumonia (FIIP) is rarely observed, and several case reports of FIIP treated with steroids have been published previously that showed the mortality is extremely high. Here, we present a 74-year-old woman who received modified
\end{abstract}


FOLFOX6 as adjuvant chemotherapy after rectal cancer surgery. The patient experienced FIIP, which improved after application of steroid pulse (high-dose methylprednisolone at 1,000 $\mathrm{mg} /$ day for 3 days) and tapering (starting with prednisolone at $40 \mathrm{mg} /$ day) therapy. Our data suggest that such a steroid therapy could represent an effective treatment option for FIIP.

(C) 2020 The Author(s).

Published by S. Karger AG, Basel

\section{Introduction}

In recent decades, the chemotherapy treatment for colorectal cancer (CRC) has undergone substantial development, including the application of effective new drugs [1,2]. Oxaliplatin with 5-fluorouracil plus l-leucovorin (FOLFOX) is used as adjuvant chemotherapy in patients with pathological stage III colon cancer (CC), and FOLFOX is also a promising chemotherapeutic regimen in treating advanced or metastatic and unresectable CRC [1-4]. In addition, the FOLFOX regimen has also been used for the treatment of advanced gastric cancer cases [5], and its indications have been expanding.

While the FOLFOX regimen is associated with adverse effects (gastrointestinal, neurosensory, and hematological toxicities), drug-induced interstitial pneumonia (DI-IP) is rarely observed in patients treated with the FOLFOX regimen. There are no established guidelines or curative treatment of DI-IP; as such, the onset of FOLFOX-induced interstitial pneumonia (FIIP) could lead to acute respiratory failure and death. Oxaliplatin has been identified as the potential causative agent of DI-IP in patients treated with FOLFOX or FOLFIRI regimens [6]. However, its mortality rate is high and its mechanism remains elusive.

Here, we present a case where a combination of steroid pulse and tapering therapy was effective in treating DI-IP related to modified FOLFOX6 (mFOLFOX6).

\section{Case Report}

A 74-year-old woman had a history of hypertension and chronic obstructive pulmonary disease. Her arterial blood gas analysis showed that the partial pressures of oxygen $\left(\mathrm{PaO}_{2}\right)$ and carbon dioxide $\left(\mathrm{PaCO}_{2}\right)$ were 65.7 and $35.8 \mathrm{~mm} \mathrm{Hg}$ on room air, respectively, which did not impact her daily life activities. She underwent Hartmann's procedure and lymph node resection for the treatment of rectal cancer in our hospital. The final pathological diagnosis was classified as pT3N2bM0 pStage IIIC in accordance with the Union for International Cancer Control (UICC) tumor node metastasis (TNM) classification.

Two months after surgery, she received mFOLFOX6 as adjuvant chemotherapy. The mFOLFOX6 therapy consisted of l-leucovorin $\left(200 \mathrm{mg} / \mathrm{m}^{2}\right)$ infusion over $2 \mathrm{~h}$ and oxaliplatin $\left(85 \mathrm{mg} / \mathrm{m}^{2}\right)$ on day 1 , followed by bolus injection of 5 -fluorouracil (5-FU) (400 mg/m $\left.{ }^{2}\right)$ and $46 \mathrm{~h}$ infusion of $5-\mathrm{FU}\left(2,400 \mathrm{mg} / \mathrm{m}^{2}\right)$. This regimen was repeated every 2 weeks, and 12 cycles of the treatment were administered.

The day after the completion of adjuvant chemotherapy she was hospitalized due to cough (day 0). Three days later, this condition deteriorated and fine crackles were heard in both lungs (day 3). The peripheral oxygen saturation $\left(\mathrm{SpO}_{2}\right)$ level measured by a pulse oximeter was $77 \%$ and her arterial blood gas analysis showed that the $\mathrm{PaO}_{2}$ and $\mathrm{PaCO}_{2}$ were 42.9 and $34 \mathrm{~mm} \mathrm{Hg}$ on room air, respectively. In both lungs, chest X-ray demonstrated diffuse ground-glass opacities (Fig. 1a) and chest computed tomography (CT) scan showed severe disorder of lung architecture including honeycombing (Fig. 1b). Laboratory data showed that the white blood cell count was $4.4 \times 103 / \mu \mathrm{L}$, C-reactive protein level was $3.01 \mathrm{mg} / \mathrm{dL}$ (normal value $<0.1 \mathrm{mg} / \mathrm{dL}$ ), lactate dehydrogenase (LDH) level was $739 \mathrm{U} / \mathrm{L}$ (normal range 120-220 

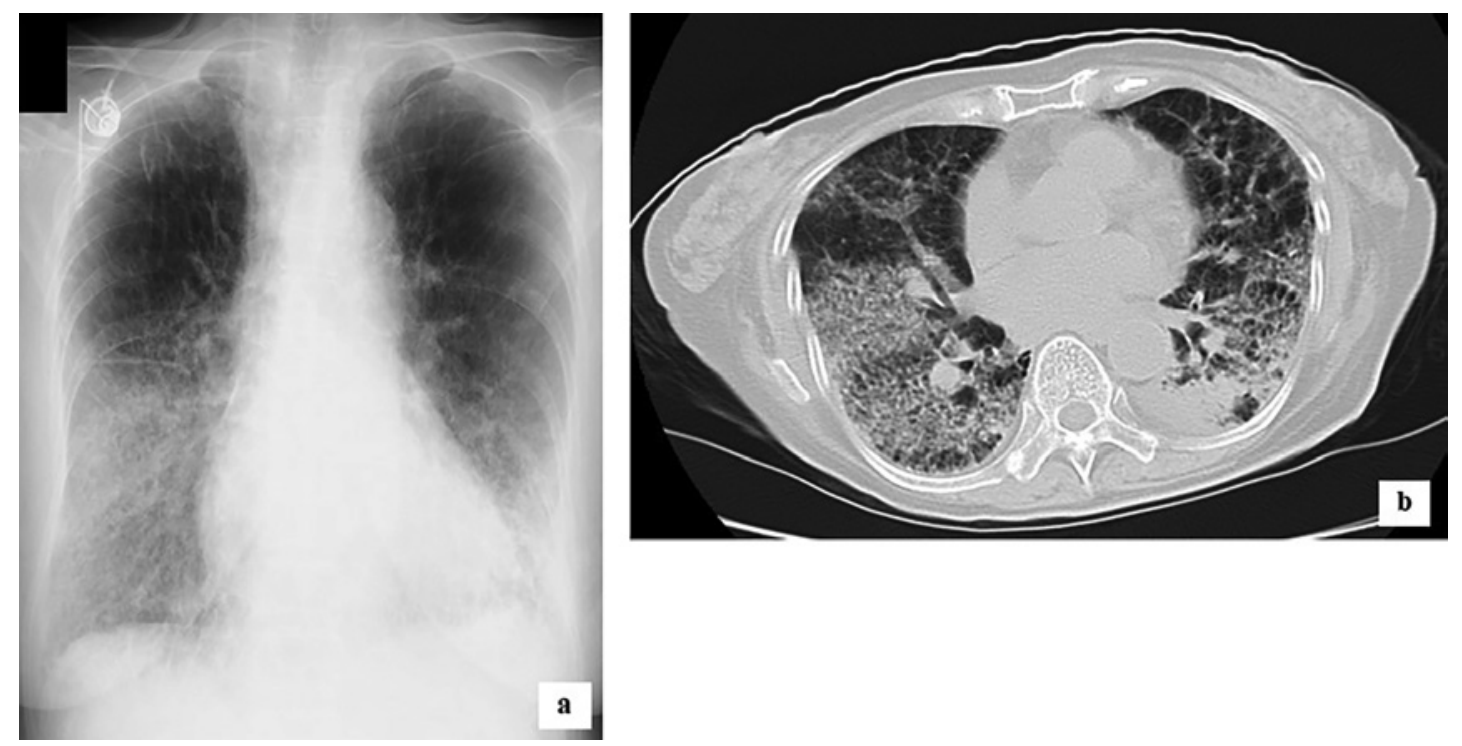

Fig. 1. Chest X-ray (a) and CT scan (b) of the patient at the onset of FIIP. a Reticular shadows with groundglass opacities could be observed mainly in the middle and lower zones of both lungs. $\mathbf{b}$ Diffuse ground-glass infiltration with honeycomb-like shadow could be observed in both lungs.
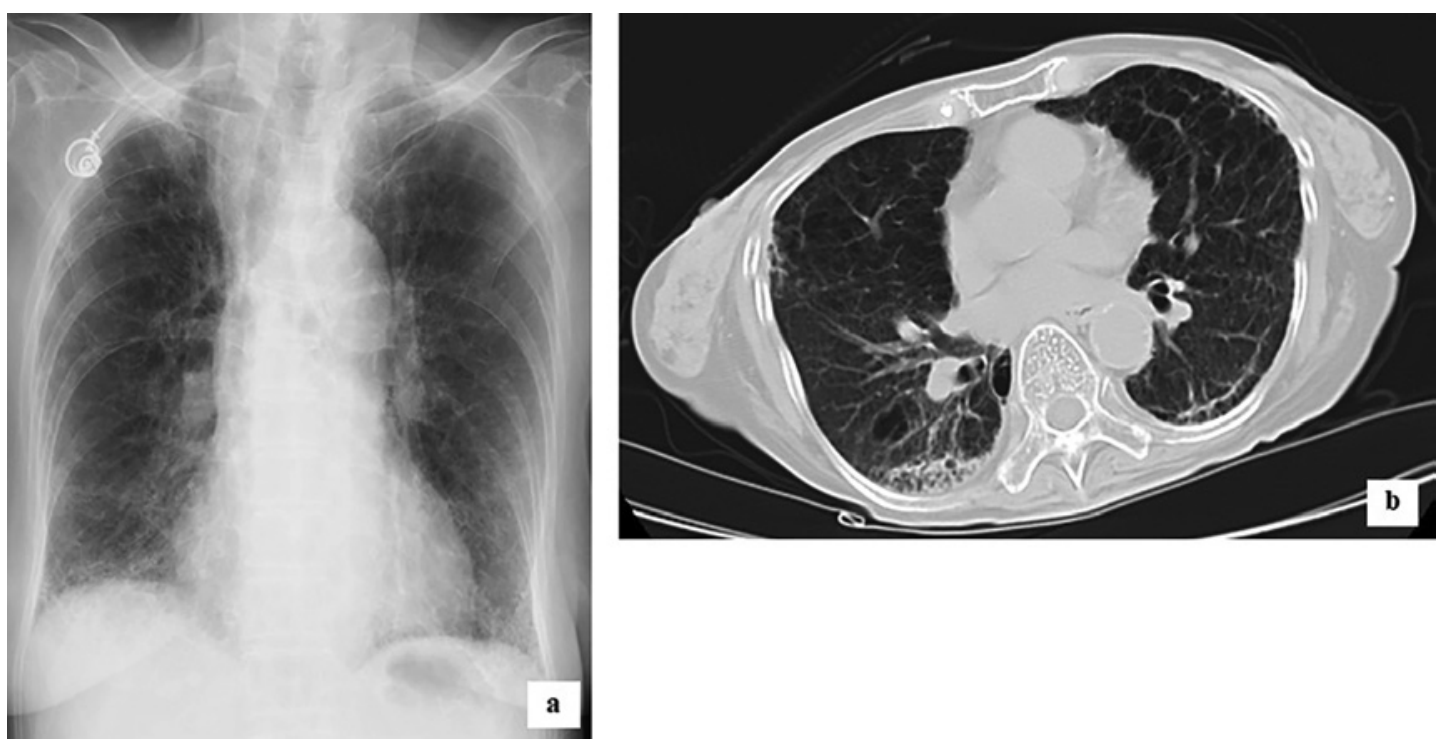

Fig. 2. Chest X-ray (a) and CT scan (b) of the patient 6 months after the completion of the steroid therapy. a In both lungs, reticular shadows with ground-glass opacities improved. b Although the old inflammation remained in the right lung, the honeycomb-like shadow had almost disappeared in both lungs.

U/L), Krebs von den Lungen-6 (KL-6) concentration was $654 \mathrm{U} / \mathrm{mL}$ (normal value $<500 \mathrm{U} /$ $\mathrm{mL}$ ), procalcitonin concentration was $<0.5 \mathrm{ng} / \mathrm{mL}$ (normal value $<0.5 \mathrm{ng} / \mathrm{dL}$ ), and $\beta$-D glucan concentration was $11.1 \mathrm{pg} / \mathrm{mL}$ (normal range $0-20 \mathrm{pg} / \mathrm{mL}$ ). All cultures and stains for infectious etiologies, including common bacteria, fungi, pneumocystis, and legionella, were negative. Although transbronchial lung biopsy (TBLB) and bronchoalveolar lavage (BAL) were not performed, we diagnosed the patient's condition as FIIP based on these examination results and clinical course. 
In addition to supplemental oxygen $(2 \mathrm{~L} / \mathrm{min})$, steroid therapy was started with a combination of broad-spectrum antibiotics (meropenem) and sivelestat sodium $(4.8 \mathrm{mg} / \mathrm{kg}$ ). Intravenous administration of steroid pulse (high-dose methylprednisolone at 1,000 mg/day for 3 days) was performed, followed by the administration of prednisolone ( $40 \mathrm{mg} /$ day) for 1 month, and then gradually decreased as the respiratory condition improved. The duration of steroid therapy was three and a half months. Subsequently, supplemental oxygen (0.5-1 L/ $\mathrm{min}$ ) and respiratory rehabilitation were continued for 4 months. In contrast, broad-spectrum antibiotics and sivelestat sodium were used for only 2 weeks.

Six months after the completion of the steroid therapy, her arterial blood gas analysis showed that the $\mathrm{SpO}_{2}$ was $92 \%$, while $\mathrm{PaO}_{2}$ and $\mathrm{PaCO}_{2}$ were 62.3 and $34.8 \mathrm{~mm} \mathrm{Hg}$ on room air, respectively, and she did not require supplemental oxygen. Her respiratory condition was almost as stable as before the rectal cancer surgery, and chest X-ray and CT scans showed significant improvements (Fig. 2a,b).

Moreover, there has been no recurrence of cancer for 20 months after the rectal cancer surgery.

\section{Discussion/Conclusion}

Chemotherapy for CRC has recently undergone substantial development, including the application of effective new drugs [1, 2]. The FOLFOX regimen has been effective as adjuvant chemotherapy in patients with stage III CC and became one of the most commonly used firstline chemotherapies for patients with unresectable or metastatic CRC [1-4].

The widely known FOLFOX-induced side effects are neurological, hematopoietic, and gastrointestinal toxicities. Pulmonary toxicities, including DI-IP, have been noted in less than $1 \%$ of CRC treated with the FOLFOX regimen during clinical trials. It is often difficult to differentiate DI-IP from other lung diseases and thus, DI-IP diagnosis remains a challenge. While TBLB or BAL may be helpful in DI-IP diagnosis, the accuracy rate of these diagnostic procedures is not high and they could only rule out other causes [7]. Steroid therapy is generally used for DI-IP; however, no prospective studies have examined the outcomes of such a therapy in these patients [7].

Although several case reports on FIIP have been published [8-14], currently, there is no established guideline for the management of FIIP. Most of the published reports on FIIP primarily focused on the application of steroid treatment. One specific report showed some improvements in FIIP with steroid pulse therapy alone [8], but other reports showed no improvements with intensive care and steroid pulse therapy [9-12].

While the pathogenesis of FIIP is unknown, oxaliplatin is considered to be the causative drug linked to FIIP in these reports [8-14]. The mechanism of oxaliplatin-induced pneumonia is not yet determined; however, data suggest that oxaliplatin could cause glutathione depletion, which could be involved in the pathogenesis of liver injury leading to hepatic sinusoidal obstruction associated with endothelial injury and perivenular fibrosis [15]. Since glutathione acts as a protector against oxidative damage in the lungs, depletion of the same caused by oxaliplatin could trigger pulmonary lesions leading to interstitial pulmonary disorder or subsequent pulmonary fibrosis. Currently, it remains elusive if treatment with antioxidant agents (such as $\mathrm{N}$-acetylcysteine) that replenish glutathione deposits could be beneficial in treating FIIP. However, since glutathione protects against oxidative damages, its supplementation could have a positive impact on FIIP patients. This hypothesis is supported by studies that showed $\mathrm{N}$-acetylcysteine, with or without steroid pulse therapy, administration improved FIIP $[13,14]$.

In our case, after the clinical diagnosis of FIIP, steroid pulse therapy (intravenous administration of high-dose methylprednisolone at $1,000 \mathrm{mg} /$ day) followed by steroid tapering 
Yanagawa et al.: Drug-Induced Interstitial Pneumonia due to FOLFOX

Fig. 3. Changes in the serum level of LDH and KL- 6 between posthospitalization day 3 and day 252 and the clinical course of steroid therapy.

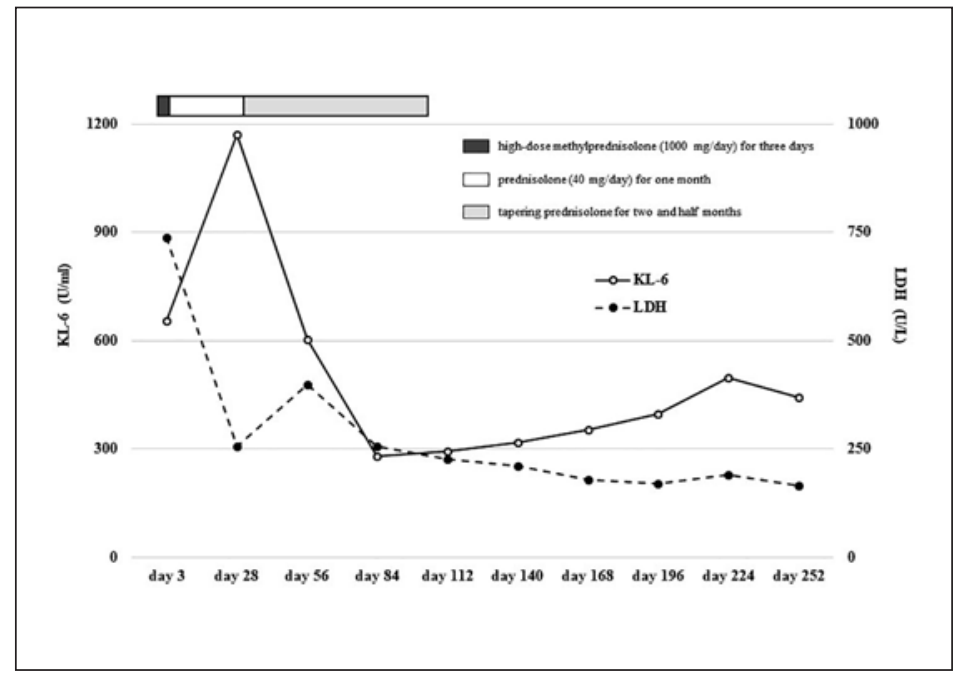

therapy (starting with prednisolone at $40 \mathrm{mg}$ /day) gradually improved the respiratory status in the patient. The steroid therapy brought the serum level of KL-6 and LDH within the normal range and this effect sustained even after the completion of the therapy (Fig. 3). Bacterial pneumonia that developed twice after the completion of steroid treatment improved rapidly with ampicillin administration.

Collectively, these data suggest that a combination of steroid pulse and tapering therapy could be an effective treatment option for FIIP, and if such a therapy is insufficient, application of antioxidants could become another treatment option.

\section{Acknowledgement}

The authors would like to thank the patient for providing consent to publish clinical information and data. We would like to thank Editage (www.editage.com) for English language editing.

\section{Statement of Ethics}

Written informed consent was obtained from the patient and her family.

\section{Conflict of Interest Statement}

The authors have no conflicts of interest to declare.

\section{Funding Sources}

The authors received no financial support for the research, authorship, or publication of this article. 
Yanagawa et al.: Drug-Induced Interstitial Pneumonia due to FOLFOX

\section{Author Contributions}

S.Y. made a substantial contribution toward the concept and design of the study and in data acquisition and interpretation. N.K., T.M., S.K., Y.T., and K.S. were involved in drafting the manuscript and critical revision of the intellectual content. S.Y. approved the final version of the manuscript submitted for publication. All authors read and approved the manuscript.

\section{References}

1 Ciombor KK, Wu C, Goldberg RM. Recent therapeutic advances in the treatment of colorectal cancer. Annu Rev Med. 2015;66:83-95.

2 Das S, Ciombor KK, Haraldsdottir S, Goldberg RM. Promising new agents for colorectal cancer. Curr Treat Options Oncol. 2018 May 11;19(6):29.

3 Reynolds J, Chamberland-Tremblay A, Herrington JD, Munoz Maldonado Y, Wong L. High- versus low-dose leucovorin in the modified FOLFOX6 regimen for first-line treatment of metastatic colorectal cancer J Oncol Pharm Pract. 2017 Apr;23(3):173-8.

4 Gustavsson B, Carlsson G, Machover D, Petrelli N, Roth A, Schmoll HJ, et al. A review of the evolution of systemic chemotherapy in the management of colorectal cancer. Clin Colorectal Cancer. 2015 Mar;14(1):1-10.

5 Masuishi T, Kadowaki, S, Kondo M, Komori A, Sugiyama K, Mitani S, et al. FOLFOX as first-line therapy for gastric cancer with severe peritoneal metastasis. Anticancer Res. 2017 Dec;37(12):7037-42.

6 Shimura T, Fuse N, Yoshino T, Minashi K, Tahara M, Doi T, et al. Clinical features of interstitial lung disease induced by standard chemotherapy (FOLFOX or FOLFIRI) for colorectal cancer. Ann Oncol. 2010 Oct;21(10): 2005-10.

7 Skeoch S, Weatherley N, Swift AJ, Oldroyd A, Johns C, Hayton C, et al. Drug-induced interstitial lung disease: a systematic review. J Clin Med. 2018 Oct 15;7(10):356.

8 Soon WC, West K, Gibeon D, Bowen EF. Pulmonary fibrosis secondary to FOLFOX chemotherapy: a case report. Case Rep Oncol. 2014 Sep 24;7(3):662-8.

9 Ishizone S, Koide N, Akita N, Karasawa F, Kobayashi N. Koizumi T, et al. Fatal interstitial pneumonia associated with oxaliplatin-based therapy in a patient with metastatic rectal cancer. Clin J Gastroenterol. 2011 Jun; 4(3): 157-61.

10 Vargas A, Montironi C, Buxó E. Organizing pneumonia with fatal outcome after adjuvant chemotherapy with FOLFOX. Arch Bronconeumol. 2015 Nov;51(11):610.

11 Moskovitz M, Wollner M, Haim N. Oxaliplatin-induced pulmonary toxicity in gastrointestinal malignancies: two case reports and review of the literature. Case Rep Oncol Med. 2015;2015:341064.

12 Sánchez Cendra C, Juez Martel I, Gutierrez Abad D. Interstitial lung disease caused by oxaliplatin. An uncommon but not unknown complication. Arch Bronconeumol. 2017 Apr;53(4):213-5.

13 Wilcox BE, Ryu JH, Kalra S, Wang S, Guo J. Exacerbation of pre-existing interstitial lung disease after oxaliplatin therapy: a report of three cases. Respir Med. 2008 Feb;102(2): 273-9.

14 De Weerdt A, Dendooven A, Snoeckx A, Pen J, Lammens M, Jorens PG. Prognosis and treatment of FOLFOX therapy related interstitial pneumonia: a plea for multimodal immune modulating therapy in the respiratory insufficient patient. BMC Cancer. 2017 Aug 29;17(1):586.

15 Rubbia-Brandt L, Audard V, sartoretti P, Roth AD, Brezault C, Le Charpentier M, et al. Severe hepatic sinusoidal obstruction associated with oxaliplatin-based chemotherapy in patients with metastatic colorectal cancer. Ann Oncol. 2004 Mar;15(3):460-6. 